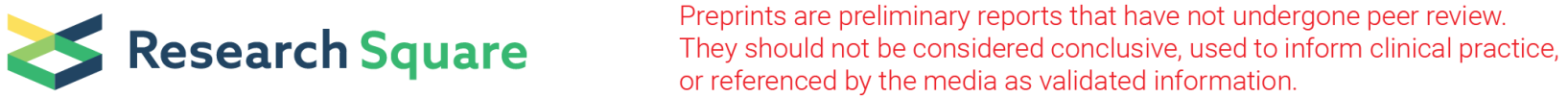 \\ Vitamin D Deficiency and Depression in Obese Adults: A Case- Control Study
}

\section{Leila Kamalzadeh}

Iran University of Medical Sciences

Atefeh Ghanbari Jolfaei ( $\nabla$ ghanbari.a@iums.ac.ir)

Iran University of Medical Sciences

Malihe Saghafi

Iran University of Medical Sciences

\section{Research Article}

Keywords: Vitamin D Deficiency, 25-hydroxyvitamin D, Obesity, BMI, Depression

Posted Date: April 14th, 2021

DOl: https://doi.org/10.21203/rs.3.rs-414214/v1

License: (c) (1) This work is licensed under a Creative Commons Attribution 4.0 International License. Read Full License 


\section{Abstract}

Background: Depression is one of the most prevalent psychiatric disorders reported in obese population. Amongst the contributing factors of depression, vitamin D deficiency has increasingly drawn attention in recent years. This paper seeks to examine the association between serum vitamin D level and depression in patients with obesity.

Methods: This case-control study included 173 depressed obese patients and 174 non-depressed controls. Structured Clinical Interview for DSM-5 (SCID-5) was used to confirm the diagnosis of depression. 25-Hydroxyvitamin D [25(OH)D], Thyroid stimulating hormone (TSH), fasting blood sugar (FBS), parathyroid hormone (PTH) levels, and BMI were assessed in both groups. The statistical analyses included T-test, Chi-squared test, and multivariable logistic regression.

Results: The mean 25(OH)D levels were significantly different between the case and control groups $(20.43 \pm 15.37$ vs. $26.55 \pm 13.17, P<.001)$. Vitamin $D$ insufficiency/deficiency was detected in $77.6 \%$ and $67.4 \%$ of the case and control groups, respectively, which was significantly different $(P=.034)$. Being female, greater age and lower vitamin $D$ levels were associated with greater odds of developing depression $(\mathrm{OR}=3.57,95 \% \mathrm{Cl}=1.82-7.02 ; \mathrm{OR}=1.05,95 \% \mathrm{Cl}=1.02-1.07 ; \mathrm{OR}=1.51,95 \% \mathrm{Cl}=1.16-1.96$, respectively).

Conclusion: The present study provides additional evidence with respect to the hypothesis that low vitamin $D$ serum concentration is associated with depression in obese adults, and highlights the need for further research to determine whether this association is causal.

\section{Introduction}

Obesity is increasingly recognized as a serious public health concern. More than 650 million people throughout the world suffer from overweight and obesity(1). There is strong evidence to suggest that obesity is conjoint with several mental and physical problems(2). Depression is one of the most prevalent psychiatric disorders reported in obese population contributing to significant disability, mortality and healthcare costs(3). The exact mechanisms linking depression and obesity have not been established. Studies have shown multiplex interactions between biologic, psychologic, and environmental factors giving rise to the association between obesity and depression(4). Amongst the contributing factors of depression, vitamin $\mathrm{D}$ deficiency has increasingly drawn attention in recent years(5).

Vitamin D, also known as cholecalciferol, is a unique neuro-steroid hormone which is vital for numerous brain functions. This hormone binds to receptors in numerous regions of the brain including the hippocampus and cingulate cortex, which are involved in the pathogenesis of depression and other mental illnesses(6). Many clinical studies have found depression, anxiety and cognitive impairment to be associated with low serum levels of 25-hydroxyvitamin $D[25(\mathrm{OH}) \mathrm{D}]$, which is the major circulating form of vitamin D, in average weight people(5-7). Recent research has also provided evidence for antidepressant properties of vitamin D supplementation $(8,9)$. 
Strong evidence of aberrations in the vitamin D-endocrine system as well as low serum 25(OH)D levels have been seen in obese individuals $(10,11)$. It has been demonstrated that vitamin $D$ deficiency is $35 \%$ more likely in obese people compared to the healthy-weight subjects(12).Volumetric dilution of Vitamin D is the most plausible mechanism of low $25(\mathrm{OH}) \mathrm{D}$ in individuals with obesity(11). Alternative mechanisms for lower 25(OH)D concentrations in patients with obesity include lower dietary consumption, decreased dermal synthesis, reduced intestinal absorption, altered metabolism, as well as less sunlight exposure due to lower physical activity(13).

In spite of a biologically potential role of vitamin $D$ in the development of depression, very few studies have investigated this association in overweight and obese subjects, with conflicting findings. While two studies indicated the benefits of vitamin D supplementation on depressive symptoms in obese adults(14, 15), a recent randomized trial presented contradictory results(16).

The discrepancy among these results has given rise to the need for further research. In this regard, this study seeks to examine the association between serum vitamin $D$ level and depression in obese patients.

\section{Methods}

\section{Study design}

This case-control study was conducted at Rasoul-e Akram hospital, an affiliate of Iran University of Medical Sciences located in Tehran, Iran.

\section{Study Participants}

The study population consisted of males and females aged 18 to 60 years old visiting out-patient obesity clinic from April 2019 to October 2020. 173 depressed patients (cases) and 174 non-depressed controls were enrolled in the study. Eligibility criteria required patients to have a BMI greater than or equal to 30 , normal thyroid function, normal fasting blood sugar, normal PTH level, no history of sleep apnea, neither past nor present substance use, no history of calcium supplement or vitamin D use. Additional criteria for the non-depressed group included: not meeting criteria for another mental disorder and no history of psychiatric medication use. Patients with incomplete medical records were excluded from the study.

\section{Measurements}

\section{Anthropometric Parameters}

Height and weight were measured and body mass index (BMI) was calculated as weight/height ${ }^{2}\left(\mathrm{~kg} / \mathrm{m}^{2}\right)$.

\section{Assessment of depression}

To confirm the diagnosis of depressive disorder, participants were interviewed by expert psychiatrists using the Structured Clinical Interview for the Diagnostic and Statistical Manual of Mental Disorders (DSM-5) (SCID-5) (17, 18). 


\section{Demographic and clinical information}

Participants' medical information was collected using hospital database which included data on basic demographics, medical and psychiatric diagnoses, history of substance and medication use, and physical and psychiatric examination, as well as laboratory data including serum levels of thyroid stimulating hormone (TSH), fasting blood sugar (FBS), and parathyroid hormone (PTH). Considered laboratory reference ranges were: TSH (0.35-5.5 mIU/L), FBS (<100 mg/dl), and PTH (0-55 pg/mL).

\section{Vitamin D Assays}

The circulating levels of 25(OH)D in the plasma samples were measured using Enzyme immunoassay (EIA) method (IDS, UK). The final measurements were classified according to definitions established by the Endocrine Society: $30-100 \mathrm{ng} / \mathrm{ml}$ was considered normal, with insufficient 25(OH)D levels or hypovitaminosis $D$ sub grouped into two: vitamin $D$ insufficiency $(20-29 \mathrm{ng} / \mathrm{ml})$ and vitamin $D$ deficiency $(<20 \mathrm{ng} / \mathrm{ml})(19)$.

\section{Statistical analysis}

The Statistical Package for Social Sciences, version 22 was used for all statistical analyses (SPSS Inc., Chicago, IL, USA). Statistical significance was analyzed using analysis of variance, T-test and Chisquared test as appropriate. Multivariable logistic regression was performed to assess the impact of confounders. A $p$-value of $<0.05$ was considered statistically significant.

\section{Results}

A total of 174 depressed patients and 173 non-depressed controls were studied. Statistically significant differences were observed among the two groups in terms of the ratio of females to males $(P<.001)$ and age $(P<.001)$, i.e., the case group had a significantly higher proportion of females $(92 \%$ vs. $75.1 \%)$ and a higher mean age than the control group $(41.76 \pm 10.016$ vs. $37.05 \pm 9.857)$. The mean vitamin $D$ levels were also significantly different between the case and control groups $(20.43 \pm 15.37$ vs. $26.55 \pm 13.17, P$ <.001). Moreover, vitamin D insufficiency/deficiency was detected in $77.6 \%$ and $67.4 \%$ of the case and control groups, respectively, which was significantly different $(P=.034)$. The mean BMI was not significantly different between the groups $(P=.051)$. The baseline characteristics of the participants are summarized in Table 1. 
Table 1

Baseline characteristics of the participants

\begin{tabular}{|llll|}
\hline Characteristic & $\begin{array}{l}\text { Cases } \\
\mathbf{N}=\mathbf{1 7 4}\end{array}$ & $\begin{array}{l}\text { Controls } \\
\mathbf{N}=173\end{array}$ & Pvalue \\
\hline Age (years) & $41.76 \pm 10.016$ & $37.05 \pm 9.857$ & $<.001$ \\
\hline Female, $\mathrm{n}(\%)$ & $160(92.0 \%)$ & $130(75.1 \%)$ & $<.001$ \\
\hline BMI $\left(\mathrm{Kg} / \mathrm{m}^{2}\right)$ & $41.93 \pm 7.42$ & $43.45 \pm 7.02$ & .051 \\
\hline Vitamin D level $(\mathrm{ng} / \mathrm{mL})$ & $20.43 \pm 15.37$ & $26.55 \pm 13.17$ & $<.001$ \\
\hline Vitamin D status, $\mathrm{n}(\%)$ & $39(22.4 \%)$ & $56(32.3 \%)$ & .034 \\
Normal & $135(77.6 \%)$ & $117(67.4 \%)$ & \\
\hline Insufficient/Deficient & & & \\
\hline
\end{tabular}

Table 2

shows the logistic regression results in which, depression is the dependent variable and age, gender and $25(\mathrm{OH}) \mathrm{D}$ are independent variables. As can be seen, vitamin D level, age, and gender are significantly associated with depression.

\begin{tabular}{|c|c|c|c|c|c|}
\hline Variable & $B$ & $\begin{array}{l}\text { Standard } \\
\text { Error }\end{array}$ & $\begin{array}{l}\text { Wald } \\
\text { Statistic }\end{array}$ & $\begin{array}{l}P \\
\text { value }\end{array}$ & OR, $95 \% \mathrm{Cl}$ \\
\hline Age & .049 & .012 & 16.985 & $<.001$ & $\begin{array}{l}1.05(1.02- \\
1.07)\end{array}$ \\
\hline Gender (Being female) & 1.275 & .344 & 13.702 & $<.001$ & $\begin{array}{l}3.57(1.82- \\
7.02)\end{array}$ \\
\hline $\begin{array}{l}\text { Vitamin D level } \\
\text { (Insufficient/deficient) }\end{array}$ & .415 & .133 & 9.783 & .002 & $\begin{array}{l}1.51(1.16- \\
1.96)\end{array}$ \\
\hline Constant & -5.308 & .916 & 33.591 & $<.001$ & .005 \\
\hline
\end{tabular}

Table 2. Multivariable logistic regression analysis including confounders of age and gender

The estimate for the effect of age was .049 (SE =.012), $\mathrm{P}<0.001$. This translates into an OR of $1.05(95 \%$ $\mathrm{Cl}=1.02-1.07)$. This means that an increase in age by 1 year increases the odds of developing depression by $5 \%$.

The estimate for the effect of being female was $1.275(\mathrm{SE}=.344), \mathrm{P}<0.001$. This translates into an OR of $3.57(95 \% \mathrm{Cl}=1.82-7.02)$. This means that obese females are at 3.57 times greater odds of developing depression.

The estimate for the effect of vitamin $D$ level was $.415(\mathrm{SE}=.133), P=.002$. This translates to an OR of $1.51(95 \% \mathrm{Cl}=1.16-1.96)$. Thus, for each unit decrease in vitamin $\mathrm{D}$ level, the odds of developing 
depression increase by a factor of 1.51.

\section{Discussion}

The findings of the present study revealed that there is an association between low 25(OH)D serum levels and incident depression in obese patients. The relation between low levels of vitamin $\mathrm{D}$ and depression was already established in normal weight individuals by past research(5-7). However, relatively few studies have evaluated this relationship in obese adults, and results have been mixed. Consistent with our findings Jorde et al.(15) and Milaneschi et al.(4) found that low serum level of vitamin $D$ is a risk factor for depression and suggested that BMI plays an important mediating role in the association between vitamin D and depression. In the same vein, Irandoust et al.(14) demonstrated that both vitamin D supplementation and physical activity have beneficial impact on depressive symptoms in obese females. Penckofer et al.(20) yielded comparable results in their study on women with type 2 diabetes. They demonstrated that vitamin D supplementation significantly ameliorated depression in the patients.

Multiple mechanisms are involved in the interaction between vitamin D deficiency, obesity and depression. It has been proposed that an inverse relationship exists between vitamin $D$ serum levels and $\mathrm{BMI}(21)$. As was pointed out in the introduction to this paper, low serum 25(OH)D in obese patients can occur for a number of reasons including insufficient vitamin $D$ consumption, increased fat or muscle mass, genotype variation in vitamin $D$ binding proteins or enzymes responsible for vitamin $D$ metabolism $(11,13)$. On the other hand, vitamin D deficiency can increase the risk of developing depression, through several biological pathways including effects on immunomodulation, cellular signaling, modulation of hypothalamic-pituitary-adrenal axis, intracellular calcium homeostasis, and production of neurotransmitters $(6,22)$ Moreover, both obesity and vitamin D deficiency lead to a chronic low-grade inflammation, which has been suggested to contribute to the development of depression(11, 23). At last, it is also possible that PTH levels contribute to the relation between vitamin $\mathrm{D}, \mathrm{BMI}$, and depression. Recent evidence suggests that both obesity and low vitamin D levels are accompanied by significantly higher PTH levels, and high PTH levels are related to depression(4).

However, more recently, literature has emerged that offers contradictory findings about these associations. In contrast to the mentioned studies, in a randomized controlled study, Mousa et al. (16) showed that depressive symptoms in obese individuals were not associated with 25(OH)D concentrations, nor did improve by Vit D supplementation. Similarly, a recent clinical interventional cohort study revealed that vitamin $D$ supplementation for six months had no significant impact on depressive symptoms, but could improve anxiety symptoms in depressed patients with vitamin D deficiency. The authors suggested that $\mathrm{BMI}$ is an important mediating factor between low serum 25(OH)D and anxiety symptoms(24).

Different findings may be attributed to the differences in the study population (different races, gender, and age groups), diverse methodology (different vitamin D supplementation doses and duration) and different baseline levels of serum 25(OH)D concentrations. Moreover, the mentioned studies applied self- 
reported psychiatric rating scales for evaluation of depression rather than a clinician-rated assessment, and therefore were prone to multiple potential biases. In addition, some of the previous studies did not adjust for potential confounders, such as thyroid dysfunction, comorbid diabetes mellitus, sleep apnea, low physical activity, and substance use that may play a role in developing depression in obese population(25).

The current study also found that obese females are at greater odds of developing depression compared to obese males. Moreover, the analyses revealed a statistically significant relationship between increased age and developing depression. These results match those observed in earlier studies $(26,27)$. Several factors may be responsible for this gender difference, including biologic aspects such as genetic features, endocrine system, functions of neurotransmitters, as well as psychosocial variables such as lower appearance satisfaction and Gender-role attitudes(26).

\section{Conclusions}

In summary, the present study provides additional evidence with respect to the hypothesis that low vitamin $D$ serum concentration is associated with depression in obese adults, and highlights the need for further research to find out whether this association is causal.

Although the present study is based on a small sample size, the results indicate that low serum vitamin $D$ level can be a modifiable risk factor for depression in obese population. The key strength of this study is the exclusion of several potential confounders such thyroid dysfunction, comorbid diabetes, hyperparathyroidism, and substance use which were not addressed by previous studies. Moreover, in the present study, the diagnosis of depression was based on standardized semi-structured interviews by expert psychiatrists rather than self-report rating scales.

\section{Abbreviations}

BMl: Body Mass Index

DSM-5: Diagnostic and Statistical Manual of Mental Disorders

EIA: Enzyme Immunoassay

FBS: Fasting Blood Sugar

25(OH)D: 25-Hydroxyvitamin D

OR: Odds Ratio

PTH: Parathyroid Hormone

SCID-5: Structured Clinical Interview for DSM-5 
SPSS: Statistical Package for Social Sciences

TSH: Thyroid Stimulating Hormone

\section{Declarations}

\section{Ethics approval and consent to participate}

The principles of the World Medical Association Declaration of Helsinki were adopted in the present study(28). This research was approved by the independent ethics committee of Iran University of Medical Sciences (IR.IUMS.REC 1395.8721215026). All patients signed informed consent statements.

\section{Consent for publication}

Written informed consent was obtained from the patients for publication of this reseach and any accompanying images. A copy of the written consent is available for review by the Editor-in-Chief of this journal.

\section{Availability of data and materials}

The datasets used and/or analyzed during the current study are available from the corresponding author on reasonable request.

\section{Competing interests}

The authors declare that they have no competing interests.

\section{Funding}

This research received no specific grant from any funding agency in the public, commercial, or not-forprofit sectors.

\section{Authors' contributions}

LK have drafted the work and substantively revised it. AGhJ have made substantial contributions to the conception of the work. MS was a major contributor in writing the manuscript. All authors read and approved the final manuscript.

\section{Acknowledgments}

We would like to thank Dr. Vahid Saeedi for the contributions to this manuscript.

\section{References}


1. World Health Organization. Obesity and overweight [Internet]. 2020 [cited 2021 Jan 19]. Available from: https://www.who.int/news-room/fact-sheets/detail/obesity-and-overweight

2. Mathieu L, Bitterlich N, Meissner F, von Wolff M, Poethig D, Stute P. Illness perception in overweight and obesity and impact on bio-functional age. Arch Gynecol Obstet. 2018;298(2):415-26.

3. Mulugeta A, Zhou A, Power C, Hyppönen E. Obesity and depressive symptoms in mid-life: a population-based cohort study. BMC Psychiatry. 2018;18(1):297.

4. Milaneschi Y, Simmons WK, van Rossum EFC, Penninx BWJH. Depression and obesity: evidence of shared biological mechanisms. Mol Psychiatry. 2019;24(1):18-33.

5. Okasha TA, Sabry WM, Hashim MA, Abdeen MS, Abdelhamid AM. Vitamin D serum level in major depressive disorder and schizophrenia. Middle East Curr Psychiatry. 2020;27(1):34.

6. Menon V, Kar S, Suthar N, Nebhinani N. Vitamin D and depression: A critical appraisal of the evidence and future directions. Indian J Psychol Med. 2020;42(1):11-21.

7. Köhnke C, Herrmann M, Berger K. Associations of major depressive disorder and related clinical characteristics with 25-hydroxyvitamin D levels in middle-aged adults. Nutr Neurosci. 2020;

8. Alghamdi S, Alsulami N, Khoja S, Alsufiani H, Tayeb HO, Tarazi FI. Vitamin D Supplementation Ameliorates Severity of Major Depressive Disorder. J Mol Neurosci. 2020;70(2):230-5.

9. Kaviani M, Nikooyeh B, Zand H, Yaghmaei P, Neyestani TR. Effects of vitamin D supplementation on depression and some involved neurotransmitters. J Affect Disord. 2020;269:28-35.

10. Zakharova I, Klimov L, Kuryaninova V, Nikitina I, Malyavskaya S, Dolbnya S, et al. Vitamin D insufficiency in overweight and obese children and adolescents. Vol. 10, Frontiers in Endocrinology. 2019. p. 103.

11. Vranić L, Mikolašević I, Milić S. Vitamin D deficiency: consequence or cause of obesity? Medicina (Kaunas). 2019 Aug 28;55(9):541. A

12. Pereira-Santos M, Costa PRF, Assis AMO, Santos CAST, Santos DB. Obesity and vitamin D deficiency: a systematic review and meta-analysis. Obes Rev. 2015 Apr 1;16(4):341-9.

13. Duan L, Han L, Liu Q, Zhao Y, Wang L, Wang Y. Effects of vitamin D supplementation on general and central obesity: results from 20 randomized controlled trials involving apparently healthy populations. Ann Nutr Metab. 2020;76(3):153-64.

14. Irandoust K, Taheri M. The effect of Vitamin D supplement and indoor Vs outdoor physical activity on depression of obese depressed women. Asian J Sports Med. 2017;8(3).

15. Jorde R, Sneve M, Figenschau Y, Svartberg J, Waterloo K. Effects of vitamin D supplementation on symptoms of depression in overweight and obese subjects: randomized double blind trial. $\mathrm{J}$ Intern Med. 2008 Dec 1;264(6):599-609.

16. Mousa A, Naderpoor N, de Courten MPJ, de Courten B. Vitamin D and symptoms of depression in overweight or obese adults: A cross-sectional study and randomized placebo-controlled trial. $\mathrm{J}$ Steroid Biochem Mol Biol. 2018;177:200-8. 
17. American Psychiatric Association. Diagnostic and statistical manual of mental disorders (5th ed.). Arlington, VA: American Psychiatric Association; 2013.

18. First MB, Williams JBW, Karg RS, Spitzer RL. User's guide to Structured Clinical Interview for Dsm-5 Disorders (Scid-5-cv): clinician version 1st. The Encyclopedia of Clinical Psychology. Washington, DC,: American Psychiatric Association Publishing; 2015.

19. Holick MF, Binkley NC, Bischoff-Ferrari HA, Gordon CM, Hanley DA, Heaney RP, et al. Evaluation, treatment, and prevention of vitamin D deficiency: an endocrine society clinical practice guideline. $J$ Clin Endocrinol Metab. 2011 Jul 1;96(7):1911-30.

20. Penckofer S, Byrn M, Adams W, Emanuele MA, Mumby P, Kouba J, et al. Vitamin D Supplementation Improves Mood in Women with Type 2 Diabetes. Campesi I, editor. J Diabetes Res. 2017;2017:8232863.

21. Vigna L, Silvia Tirelli A, Grossi E, Turolo S, Tomaino L, Napolitano F, et al. Directional relationship between vitamin $D$ status and prediabetes: a new approach from artificial neural network in a cohort of workers with overweight-obesity. J Am Coll Nutr. 2019;38(8):681-92.

22. Lerner PP, Sharony L, Miodownik C. Association between mental disorders, cognitive disturbances and vitamin D serum level: Current state. Clin Nutr ESPEN. 2018;23:89-102.

23. Abiri B, Vafa M. Effects of vitamin D and/or magnesium supplementation on mood, serum levels of BDNF, inflammatory biomarkers, and SIRT1 in obese women: a study protocol for a double-blind, randomized, placebo-controlled trial. Trials. 2020;21(1):225.

24. Zhu C, Zhang Y, Wang T, Lin Y, Yu J, Xia Q, et al. Vitamin D supplementation improves anxiety but not depression symptoms in patients with vitamin D deficiency. Brain Behav. 2020;10(11).

25. González-Muniesa P, Mártinez-González M-A, Hu FB, Després J-P, Matsuzawa Y, Loos RJF, et al. Obesity. Nat Rev Dis Prim. 2017;3(1):17034.

26. Weinberger N-A, Kersting A, Riedel-Heller SG, Luck-Sikorski C. The relationship between weight status and depressive symptoms in a population sample with obesity: The mediating role of appearance evaluation. Obes Facts. 2018;11(6):514-23.

27. Xu Q, Anderson D, Lurie-Beck J. The relationship between abdominal obesity and depression in the general population: A systematic review and meta-analysis. Obes Res Clin Pract. 2011;5(4):e267-78.

28. World Medical Association. World medical association declaration of Helsinki: ethical principles for medical research involving human subjects. JAMA. 2013 Nov 27;310(20):2191-4. 